\title{
Basic study on active noise barrier
}

\author{
Shiro Ise, Hiroo Yano, and Hideki Tachibana \\ Institute of Industrial Science, University of Tokyo, \\ 7-22-1, Roppongi, Minato-ku, Tokyo, 106 Japan
}

(Received 17 July 1991)

\begin{abstract}
As a trial to improve the performance of passive noise barriers by applying the active control technique, basic theoretical and experimental investigation has been made. At first, the basic conception of the "active noise barrier" is presented, in which an active control system composed of a noise detector, an error sensor, a secondary sound source and an adaptive signal processing unit is attached to a passive barrier. As a preliminary study, a simple numerical calculation was performed according to the Fresnel-Kirchhoff diffraction theory, and the adaptability of the active control has been basically confirmed. Next, 1/2 scale model experiments were performed in a hemi-anechoic room to investigate the effectiveness of the active noise barrier. In this experimental study, the method combining the "filtered $x$ LMS" algorithm and the "block processing technique" was applied as the adaptive signal processing technique. As a result, it has been found that the active noise barrier is effective for sound attenuation in low frequencies.
\end{abstract}

Keywords: Active noise control, Noise barrier, Adaptive processing

PACS number: 43. 50. Ki, 43. 50. Gf

\section{INTRODUCTION}

For the aim of noise reduction, such passive control as sound insulation by walls and barriers and sound absorption by various kinds of materials are the most basic and important technologies. As a general tendency, however, they have deficiencies in low frequencies. On the other hand, the active control technologies which have been recently developed are, in general, effective for sound control in low frequencies. Therefore, it seems to be advantageous to combine these two technologies for noise control over a wide frequency range.

As an example of such an idea, we have made a basic study on the application of active noise control to passive noise barrier. ${ }^{1,2)}$ In this paper, the general idea of "active noise barrier" and basic numerical and experimental investigations are presented.

\section{PRINCIPLE OF ACTIVE NOISE BARRIER}

Figure 1 shows the basic system of the active noise barrier schematically. In this system, a noise detector microphone is located near the primary noise source and an error sensor microphone is located in the opposite side of the barrier. As the secondary sound source, a loudspeaker is set near the top of the barrier as shown in Fig. 1 to make it close to the sound diffracting path. ${ }^{3)}$ In this situation, the adaptive FIR filter is adjusted so that the sound pressure at the error sensor become minimum. As a result, the noise in a certain area in the opposite side of the barrier is expected to be reduced.

Under the condition mentioned above the transfer functions between each couple of the elements are expressed as follows.

$$
\begin{aligned}
S_{D}(\omega)= & G_{S D}(\omega) \cdot S_{S}(\omega)+G_{A D}(\omega) \cdot S_{A}(\omega) \\
& S_{A}(\omega)=H(\omega) \cdot S_{D}(\omega) \\
S_{E}(\omega)= & G_{S E}(\omega) \cdot S_{S}(\omega)+G_{A E}(\omega) \cdot S_{A}(\omega) \\
S_{P}(\omega)= & G_{S P}(\omega) \cdot S_{S}(\omega)+G_{A \mathrm{P}}(\omega) \cdot S_{A}(\omega)
\end{aligned}
$$

where

$G_{X Y}(\omega)$ : the transfer function from $X$ to $Y$ $S_{Z}(\omega)$ : the signal at the point $Z$ 


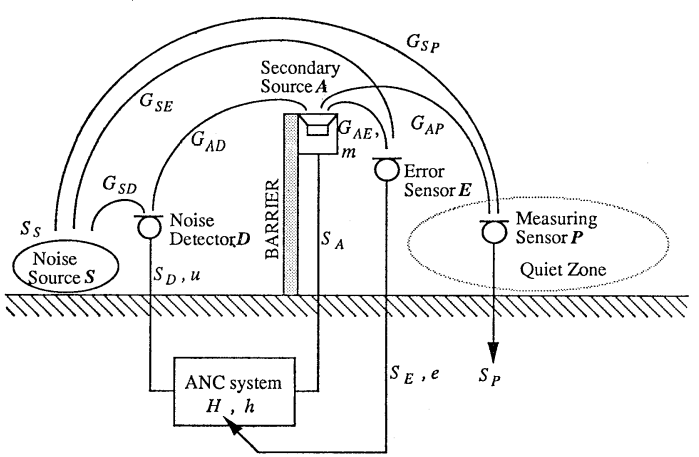

Fig. 1 Schematic diagram of the active noise barrier.

$H(\omega)$ : the transfer function of the active control system

$S:$ the noise source, $A$ : the secondary source, $D$ : the noise detector, $E$ : the error sensor, $P$ : the observation point

In Eq. (4), the second term of the right side is zero when the active control system is OFF. If the sound pressure at the error sensor can be made zero $\left(S_{E}(\omega)\right.$ $=0)$, we have the following equation from Eqs. (1), (2) and (3).

$$
H(\omega)=\frac{-G_{S E}(\omega)}{G_{S D}(\omega) \cdot G_{A E}(\omega)-G_{S E}(\omega) \cdot G_{A D}(\omega)}
$$

In this equation, the active control system becomes unstable when the denominator is small or zero. To avoid such cases, the feed back term from the secondary source to the noise detector $\left(G_{A D}(\omega)\right)$ must be small and the noise detector should be located closely to the noise source as much as possible.

The effectiveness of the active control system, the sound pressure decrease by the control, at the observation point is expressed as follows.

$$
\begin{aligned}
K & =-20 \log \left|\frac{S_{P}(\omega)}{G_{S \mathrm{P}}(\omega) \cdot S_{S}(\omega)}\right| \\
& =-20 \log \left|1+\frac{G_{A P}(\omega) \cdot S_{A}(\omega)}{G_{S P}(\omega) \cdot S_{S}(\omega)}\right|
\end{aligned}
$$

Here, by substituting Eqs. (1), (2) and (4) into Eq. (6), the following expression is obtained.

$$
K=-20 \log \left|1-\frac{G_{A P}(\omega) \cdot G_{S E}(\omega)}{G_{S P}(\omega) \cdot G_{A E}(\omega)}\right|
$$

\section{NUMERICAL STUDY}

In advance of the experimental study, the function of the active noise barrier was investigated by

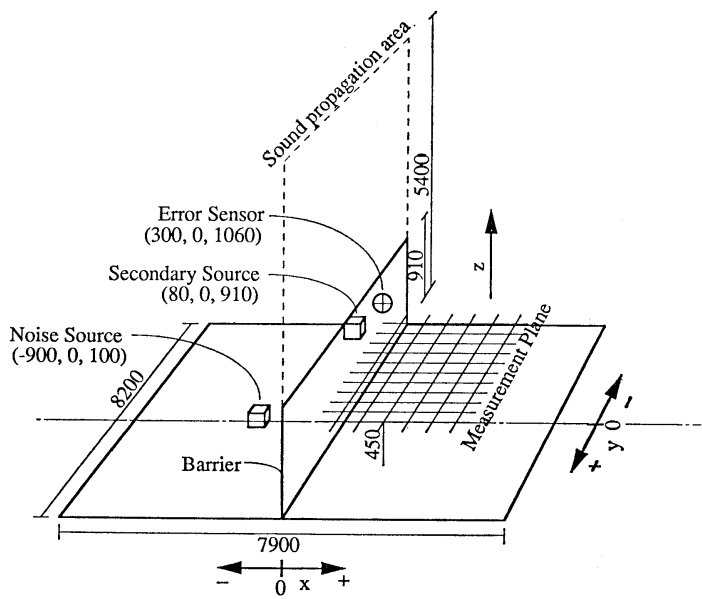

Fig. 2 Geometrical situation for the calculation.

a simple numerical calculation based on the relationships mentioned above and the Fresnel-Kirchhoff diffraction theory.

\subsection{Conditions for Calculation}

In this numerical study, the following conditions were assumed to simplify the calculation. The primary and secondary sound sources are omnidirectional point sources. The barrier has a knife-edge without thickness and its reflections are negligible. The ground surface is perfectly reflective and its reflections can be treated by the image method.4) As the geometrical situation, the following conditions were assumed (see Fig. 2).

The noise source: $(-900,0,100)$

The secondary source: $(80,0,910)$

The error sensor: $(300,0,1,060)$

The observation points (66 points): $(i, j, 450)$

( $i:-2,250$ to $2,250, j: 450$ to 2,700 , at 450 intervals)

The sound diffraction area: $(x=0,-4,100<y$ $<4,100,910<z<5,400$ ) (Unit: in $\mathrm{mm}$ )

Under these conditions, the transfer function from the noise source to the observation point $G_{S P}(\omega)$ and that from the noise source to the error sensor $G_{S E}(\omega)$ are expressed as follows.

$$
G_{S P}(\omega)=D_{S P}(\omega)+D_{S^{\prime} P}(\omega)+D_{S P^{\prime}}(\omega)+D_{S^{\prime} P^{\prime}}(\omega)
$$

$$
G_{S E}(\omega)=D_{S E}(\omega)+D_{S^{\prime} E}(\omega)+D_{S E^{\prime}}(\omega)+D_{S^{\prime} E^{\prime}}(\omega)
$$




\section{S. ISE et al.: ACTIVE NOISE BARRIER}

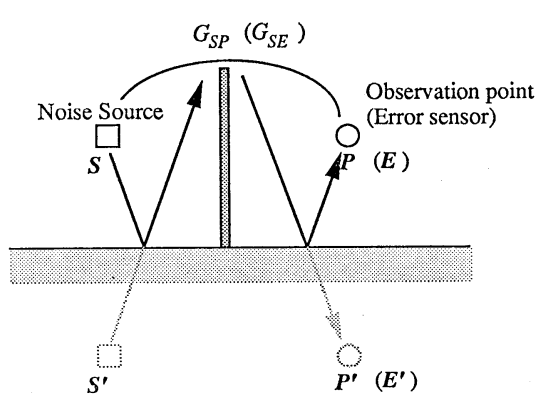

(a) $G_{S P}(\omega), G_{S E}(\omega)$

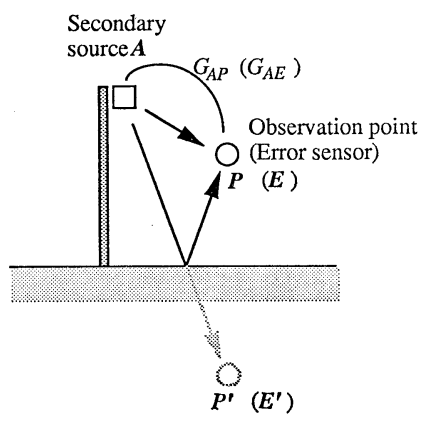

(b) $G_{A P}(\omega), G_{A E}(\omega)$

Fig. 3 Sound propagation model for the calculation.

where, $S^{\prime}, P^{\prime}$ and $E^{\prime}$ are the mirror images of $S, P$ and $E$ by the reflective ground, respectively (see Fig. 3(a)). $D_{X Y}(\omega)$ is the transfer function from $X$ to $Y$ under the assumption of the sound diffraction over a semi-infinite barrier, and it can be calculated by the following Fresnel-Kirchhoff diffraction equation.

$$
\begin{aligned}
D_{S P}(\omega)= & -\frac{j \omega}{4 \pi c} \iint_{Q} \frac{1}{r_{S Q} \cdot r_{P Q}}\left(\frac{r_{S B}}{r_{S Q}}+\frac{r_{P B}}{r_{P Q}}\right) \\
& \cdot \exp \left(-j \omega \frac{\left(r_{S Q}+r_{P Q}\right)}{c}\right) d q
\end{aligned}
$$

where,

$Q:$ the sound propagation area over the barrier

$d q$ : small area on $Q$

$r_{X Q}\left(r_{Y Q}\right)$ : the distance between $X(Y)$ and $d q$

$r_{X B}\left(r_{Y B}\right):$ the direct distance between $X(Y)$ and the barrier

$c$ : the sound velocity

The transfer function from the secondary source to the error sensor $G_{A E}(\omega)$ and that from the secondary source to the observation point $G_{A P}(\omega)$ are expressed as follows.

$$
\begin{aligned}
& G_{A E}(\omega)=F_{A E}(\omega)+F_{A E^{\prime}}(\omega) \\
& G_{A P}(\omega)=F_{A P}(\omega)+F_{A P^{\prime}}(\omega)
\end{aligned}
$$

where, $P^{\prime}$ is the mirror image of $\boldsymbol{P}$ (see Fig. 3(b)). $F_{X Y}(\omega)$ is the transfer function from $X$ to $Y$ in a free field and is expressed as follows.

$$
F_{S P}(\omega)=\frac{1}{r_{S P}} \exp \left(-j \omega \frac{r_{S P}}{c}\right)
$$

By calculating $G_{S P}(\omega), \quad G_{A P}(\omega), \quad G_{S E}(\omega)$ and $G_{A E}(\omega)$ from Eqs. (8) to (13), and substituting them into Eq. (7), the effectiveness of the active control at the observation point can be obtained.

\subsection{Results of the Calculation}

In the manner mentioned above, the effectiveness at each observation point ( 66 points in total) on a horizontal plane $0.45 \mathrm{~m}$ above the ground was calculated. The calculation was made for every $6 \mathrm{~Hz}$ and the results were combined in each $1 / 3$ octave band.

Figure 4 shows the effectiveness in each $1 / 3$ octave band from $125 \mathrm{~Hz}$ to $1 \mathrm{kHz}$. The values in the figures indicate the effectiveness (in $\mathrm{dB}$ ) of the active control, and the shadowed zones are the areas in which the effectiveness is positive, that is, the sound pressure level is decreased by the active control. In these results, the tendencies are observed that the effectiveness is relatively large and the effective area (shadowed zone) is wide in low frequency bands, especially in $125 \mathrm{~Hz}$ and $250 \mathrm{~Hz}$ bands, whereas the dangerous area in which the effectiveness is negative becomes larger in higher frequency bands.

\section{EXPERIMENTAL STUDY}

In order to investigate the effectiveness of the active noise barrier by physical experiment, 1/2 scale model study was performed as follows.

\subsection{Experimental Conditions}

Figure 5 shows the geometry for the experiments. A barrier wall of $69 \mathrm{~mm}$ in total thickness, $0.91 \mathrm{~m}$ height and $7.9 \mathrm{~m}$ length, made of two leaves of gypsum boards ( $12 \mathrm{~mm}$ thick) filled with glass fiber mat, was set in a hemi-anechoic room. As the noise source, a loudspeaker was set upward on the floor and a broad band noise was radiated from it. Another loudspeaker was attached to the top of the barrier upward as the secondary source. Regarding 


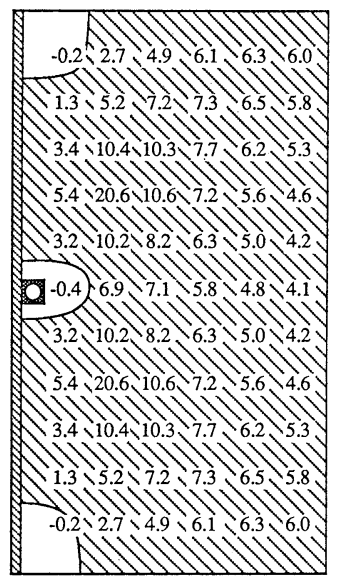

(a) $125 \mathrm{~Hz}$

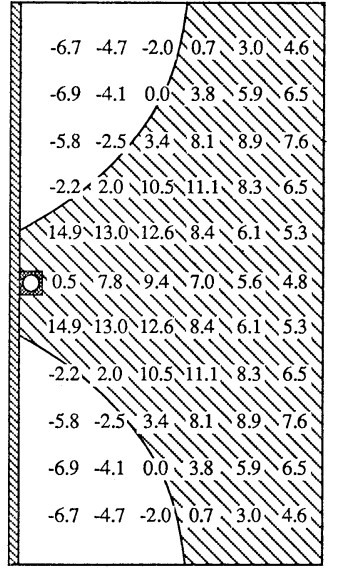

(b) $250 \mathrm{~Hz}$

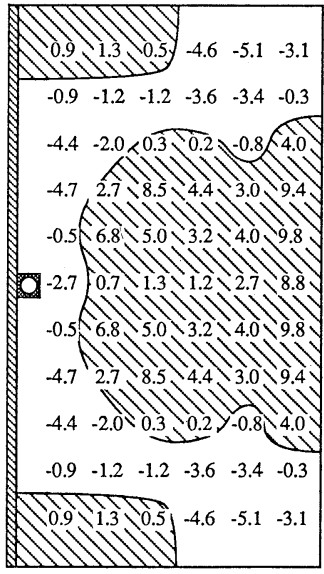

(c) $500 \mathrm{~Hz}$

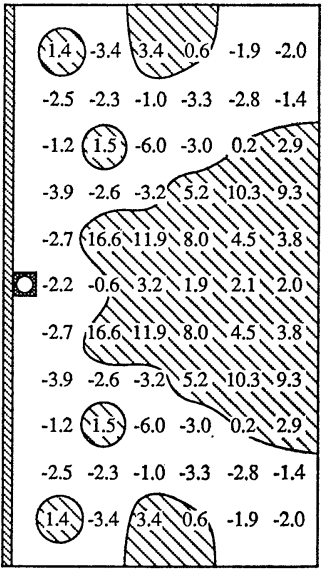

(d) $1 \mathrm{kHz}$

Fig. 4 Effectiveness obtained by the calculation.
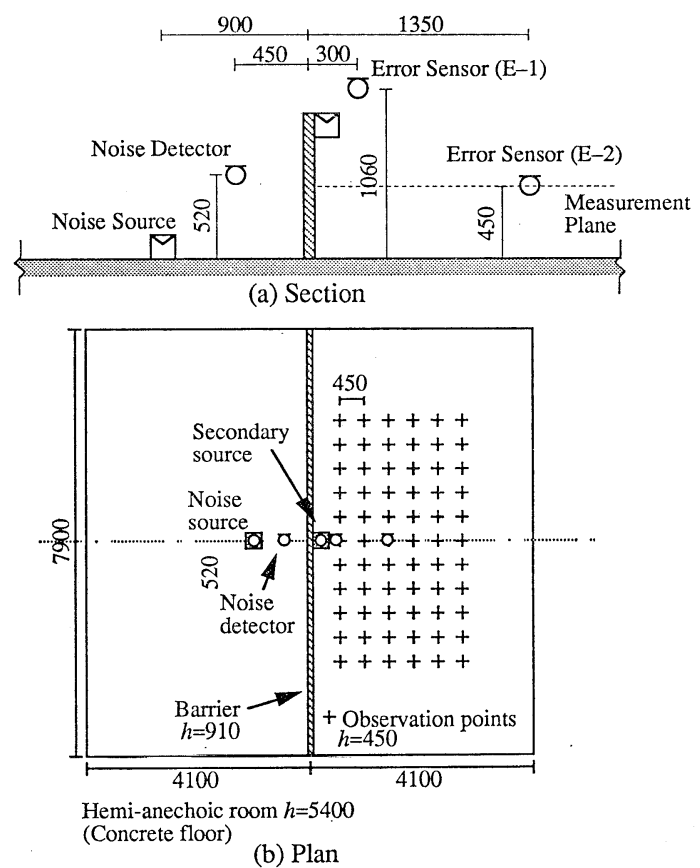

Fig. 5 Geometry for the experiments conducted in a hemi-anechoic room.

the noise detector, it should be located closely to the noise source as much as possible for the signal processing as previously mentioned. Such a situation, however, can not necessarily be allowed in actual situations. Therefore, the noise detector (an electret condenser microphone) was located at almost the midpoint between the noise source and the barrier as shown in Fig. 5. Regarding the error sensor, two positions were chosen and the difference of the effectiveness by the active control was examined as mentioned below.

\subsection{Adaptive Signal Processing}

Equation (5) means that if the transfer functions from each sound source to each sensor are known, the system of the active control can be determined. In almost actual cases, however, it is not easy to determine them and the environmental conditions are usually time-varying. In such cases, it is much advantageous to adopt the adaptive processing method. According to this method, the system minimizes the square average of the output signal from the error sensor continuously by its self-adjusting function, and therefore, the system can be robust against the change of the environmental conditions.

For the adaptive processing, the "filtered $x$ LMS algorithm"5) is well known and we also used this method in this study. In this method, the coefficients of the FIR filter are to be determined in every sampling period, but this processing can not be performed in our digital processing system. Therefore, we applied the "block processing technique"6) to the "filtered $x$ LMS algorithm" as expressed by Eq. (14).

$$
\begin{aligned}
h[l, n+1]= & h[l, n]-\mu \cdot \sum_{t=0}^{s}[e[n-t] \\
& \cdot(u[n-t-l] \otimes m)] \quad\left(l=0-S_{h}-1\right)
\end{aligned}
$$


where,

$h[l, n]: l$-th coefficient of the adaptive FIR filter at $n$-th updating

$s$ : block size of updating

$\mu$ : gain constant in adaptation

$e:$ output signal of the error sensor

$u$ : output signal of the noise detector

$m$ : impulse response from the secondary source to the error sensor

$\otimes:$ convolution integral

$S_{h}$ : tap length of the adaptive FIR digital filter

Here, $m$ must be previously known in this algorithm and therefore it must be measured before the adaptation of the system. In order to make the coefficients of the FIR filter converge, the following condition must be satisfied.

$0<\mu<\frac{2}{S_{l} \cdot r} \quad r:$ the mean-square value of $(u \otimes m)$.

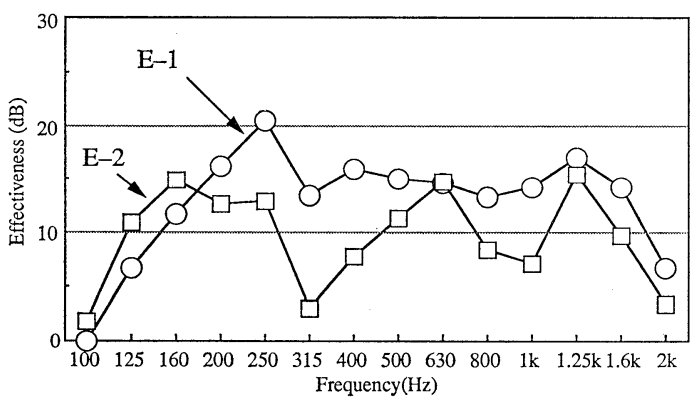

Fig. 6 Effectiveness measured at the error sensor positions.

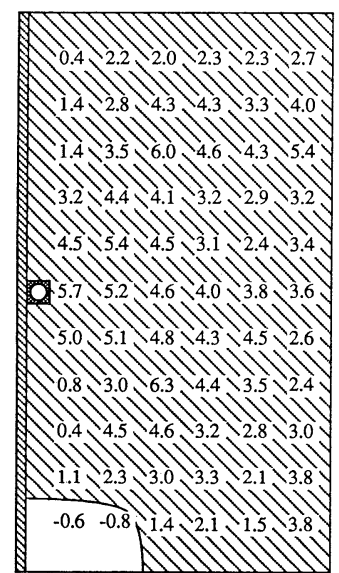

(a) $125 \mathrm{~Hz}$

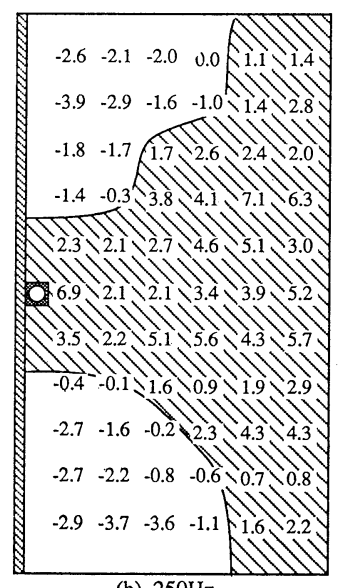

(b) $250 \mathrm{~Hz}$

For the signal processing in this study, a 32-bits personal computer (Macintosh-II) for the control system and DSP boards of our own making (processor: Motorola DSP56200, total tap length: 16,384, maximum sampling frequency: $37 \mathrm{kHz}$ ) were used. The coefficients of the FIR filter of the tap length of 1,024 calculated in the computer were transferred into the DSP boards, and the convolution calculation was performed in it in real time. The sampling frequency in $\mathrm{A} / \mathrm{D}$ and $\mathrm{D} / \mathrm{A}$ conversions was $12 \mathrm{kHz}$.

To keep the system stable, small value is needed for $\mu$. In this case, however, it takes a long time to determine the coefficients of the FIR filter. Therefore, the value of $\mu$ was determined experimentally.

\subsection{Experimental Results}

In the first experiment (Experiment-I), the error sensor was located at a high position near the secondary source (E-1 point in Fig. 5) in the same condition as in the numerical study shown in section 3.1. As the experimental results, Figure 6 shows the effectiveness of the active control, that is, the sound pressure level decrease by the control, measured at the error sensor position. In this result, effectiveness of about 10 to $20 \mathrm{~dB}$ are seen in the frequency range from $160 \mathrm{~Hz}$ to $1.6 \mathrm{kHz}$.

Figure 7 shows the effectiveness in $1 / 3$ octave bands with center frequencies of $125 \mathrm{~Hz}$ to $1 \mathrm{kHz}$ observed in the measurement plane. In these results, we can see almost the same tendencies as in the results of the numerical study, that is, the lower the center frequency is, the larger the effective area

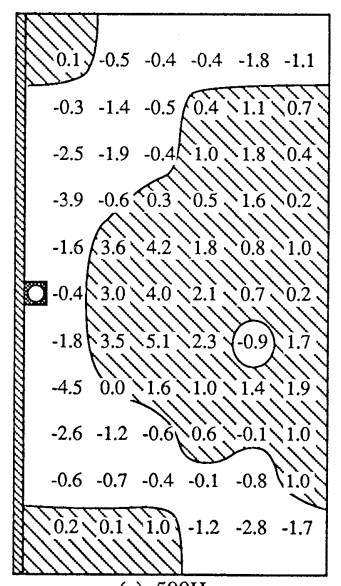

(c) $500 \mathrm{~Hz}$

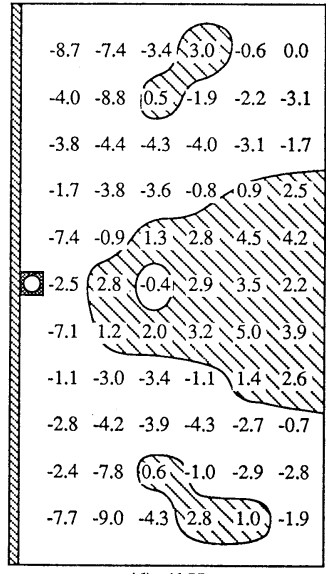

(d) $1 \mathrm{kHz}$

Fig. 7 Effectiveness observed in the measurement plane (Experiment-I). 
becomes, and the effectiveness is relatively larger in low frequency bands. This fact can be easily understood when considering that the lower the frequency is, the longer the wavelength becomes, and therefore, the wider the interference area becomes.

The shapes of the effective areas shown in Fig. 4 (numerical study) and Fig. 7 (Experiment I) are similar, but some discrepancies are seen in the absolute values of the effectiveness. This fact might be attributed to the difference of the conditions between the numerical calculation and the experiment. In the numerical study, omnidirectional characteristics were assumed for the original noise source and the secondary source, and the thickness of the barrier and its reflection were neglected in the numerical study. Besides, an ideal system which could make the sound pressure zero at the error sensor was assumed. On the other hand, such ideal conditions could not be realized in the actual experiment be- cause of the law of causality, the limitation of the tap length of the FIR filter, and the other practical conditions.

In this experiment, the sound intensity measurement was performed in a plane perpendicular to the barrier including the primary and the secondary sources, by using an intensity probe composed of a couple of $1 / 2$ inch condenser microphones. Figure 8 shows two examples of the measured results in the form of intensity vector map. In $125 \mathrm{~Hz}$ band, we can see that the vectors point to the secondary source, that means the secondary source is a negative source (sink). On the other hand, in $250 \mathrm{~Hz}$ band, it is clearly seen that the secondary source is radiating sound power, but the intensity vectors above the barrier point upward and accordingly the absolute intensities in the area behind the barrier have become small by the active control.

Next, the second experiment (Experiment-II) was
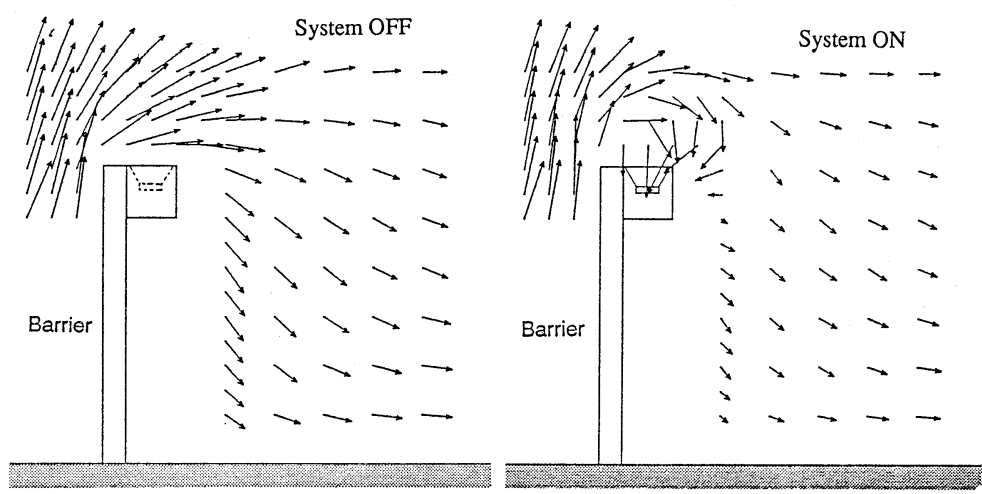

(a) $125 \mathrm{~Hz}$ in $1 / 3$ octave band
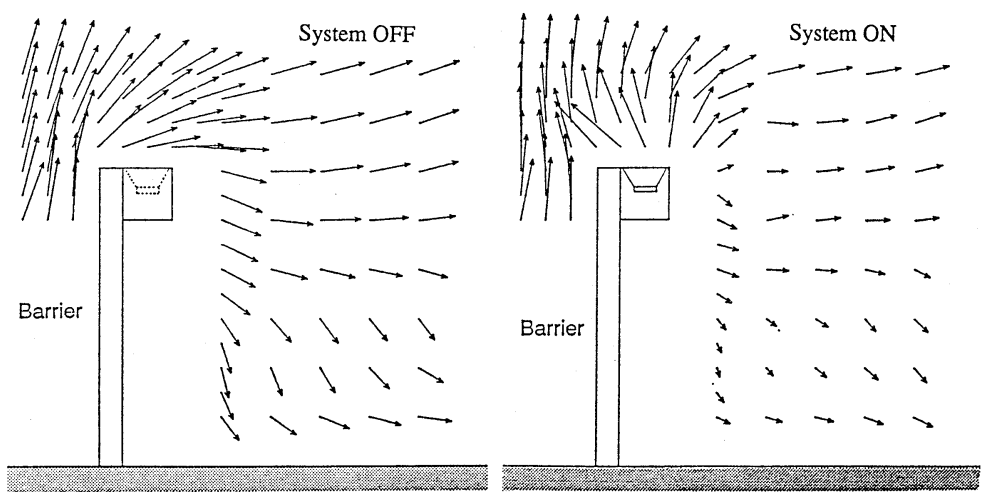

(b) $250 \mathrm{~Hz}$ in $1 / 3$ octave band

Fig. 8 Intensity vector maps around the secondary source. 


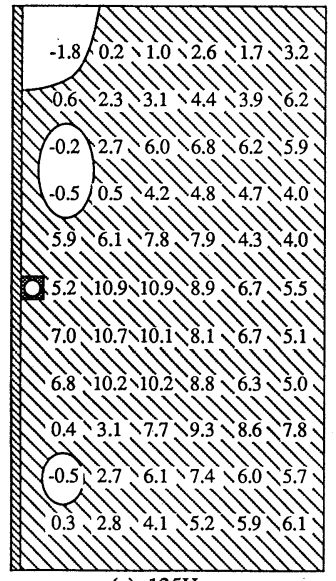

(a) $125 \mathrm{~Hz}$

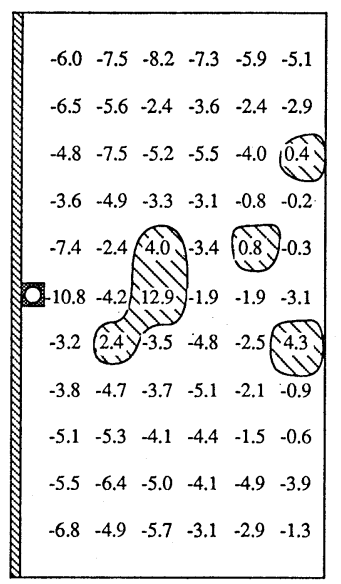

(b) $250 \mathrm{~Hz}$

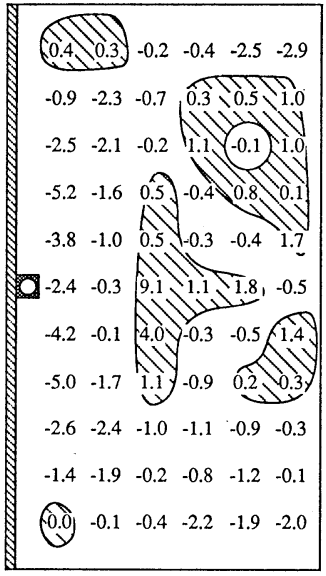

(c) $500 \mathrm{~Hz}$

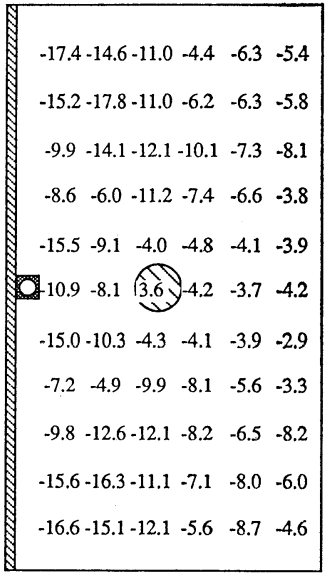

(d) $1 \mathrm{kHz}$

Fig. 9 Effectiveness observed in the measurement plane (Experiment-II).

performed by locating the error sensor on the measurement plane (E-2 point in Fig. 5) in order to see the change of effectiveness when the error sensor is within the target area.

The effectiveness at the error sensor position observed in this experiment is shown in Fig. 6 together with the result of Experiment-I. In this case, the effectiveness was lower than the former case in the frequencies higher than $200 \mathrm{~Hz}$. This fact might be explained as follows. In the case of Experiment-I, the transfer function $G_{A E}(\omega)$ was determined mainly by the direct sound from the secondary source to the error sensor. On the other hand, in the case of Experiment-II, the interference between the direct sound and the ground reflection caused peaks and dips in the transfer function, and therefore the active control system became unstable and did not converge within the tap length of the FIR filter.

Figure 9 shows the effectiveness observed on the measurement plane. In this case, a wide effective area is seen only in $125 \mathrm{~Hz}$ band and the dangerous area has expanded in other frequency bands as compared with the results of Experiment-I. The reason for this might be as follows. In Experiment-II, relatively bigger sound power was needed for the secondary source to cancel the sound from the primary noise source at the error sensor position because the error sensor was within the target area, far from the secondary source, and accordingly, sound pressure increase might appear at many points on the measurement plane. These results suggest that the error sensor should be located near the secondary source behind the barrier.

\section{CONCLUSIONS}

In this study, the possibility of the active noise barrier has been investigated by basic numerical simulation and physical experiments. As a result, it has been found that sound insulation by barrier can be improved in low frequencies by applying the active control technique. However, it has also been found that some areas with negative effectiveness can appear in higher frequencies. In order to avoid such a disadvantage and to make this kind of noise control technique effective and reliable, further investigations should be performed regarding the ways of noise detection and positioning of the secondary source and the error sensor. In this study, only one channel active control was examined. We are planning to make further investigations by adopting multi-channel active control technique ${ }^{7)}$ and directional secondary sound sources.

\section{ACKNOWLEDGEMENT}

The authors are deeply indebted to Ono-sokki Company for allowing us to use their hemi-anechoic room for the experiments in this study.

\section{REFERENCES}

1) S. Ise, N. Kanai, H. Yano, and H. Tachibana, "A trial of active control on noise barrier," Proc. Autumn Meet. Acoust. Soc. Jpn., 593-594 (1989) (in Japanese).

2) S. Ise, H. Yano, and H. Tachibana, "Appliaction 
of active control to noise barrier," Proc. 1991 Acoust. Soc. Jpn. Int. Symp. Active Control of Sound and Vibration, 309-314 (1991).

3) A. Omoto and K. Fujiwara, "The basic research of active control type noise barrier," Proc. Autumn Meet. Acoust. Soc. Jpn., 399-400 (1990) (in Japanese).

4) T. Matui, K. Takagi, K. Hiramatsu, and T. Yamamoto, "Outdoor sound propagation from a source having dimensions," J. Acoust. Soc. Jpn. (J) 45, 512-518 (1989) (in Japanese).

5) B. Widrow and S. D. Stearns, Adaptive Signal Processing (Prentice-Hall, Englewood Cliffs, 1985).

6) G. A. Clark, S. K. Mitra, and S. R. Parker, "Block implementation of adaptive digital filters," IEEE Trans. Circuits Syst. CAS-28, 584-592 (1981).

7) S. J. Elliott, I. M. Stothers, and P. A. Nelson, "A multiple error LMS algorithm and its application to the active control of sound and vibration," IEEE Trans. Acoust. Speech Signal Process. ASSP-35, 1423-1434 (1987).

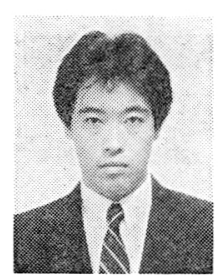

Shiro Ise Born on Mar. 131961 in Tokyo, Japan. Graduated from Waseda University in 1984, and received Engineering Doctor from Tokyo University in 1991. Researcher of Institute of Industrial Science, Tokyo University.

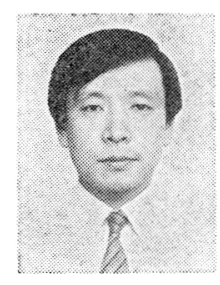

Hiroo Yano Born on Feb. 191952 in Tokyo, Japan. Graduated from Chuo University in 1975, and received Engineering Doctor from Tokyo University in 1985. Research Assistant of Institute of Industrial Science, Tokyo University.

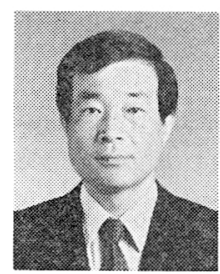

Hideki Tachibana Born on Aug. 23 1943 in Tokyo, Japan. Graduated from Tokyo University in 1967, and received Engineering Doctor from Tokyo University in 1973. Professor of Institute of Industrial Science, Tokyo University. Head of the Applied Acoustic Engineering Laboratory. A Vice-President of the Acoustical Society of Japan. 